\title{
SLC63 Gene Polymorphisms is Associated with Striatal Dopamine Transporter Changes after Glucose Loading
}

Kyoungjune Pak ( $\square$ ilikechopin@me.com )

Pusan National University Hospital

\section{Seongho seo}

Pai Chai University

Keunyoung kim

PNU: Pusan National University

Myung Jun Lee

PNU: Pusan National University

Seong-Jang kim

PNU: Pusan National University

In Joo kim

PNU: Pusan National University

\section{Research Article}

Keywords: Dopamine plasma membrane transport proteins, Genetic polymorphism, Glucose

Posted Date: May 5th, 2021

DOl: https://doi.org/10.21203/rs.3.rs-470750/v1

License: (9) (i) This work is licensed under a Creative Commons Attribution 4.0 International License. Read Full License 


\section{Abstract}

We investigated the association between SLC6A3 gene polymorphisms and changes in dopamine transporter (DAT) availability after glucose loading in humans. An intravenous injection of ${ }^{18} \mathrm{~F}-\mathrm{FP}-\mathrm{CIT}$ was administered after infusion of glucose or placebo, and the emission data were acquired over 90 min in 38 healthy male participants. DAT availability expressed in terms of binding potential $\left(\mathrm{BP}_{\mathrm{ND}}\right)$ was recorded. The 40-bp variable number of tandem repeats (VNTR) in the $3^{\prime}$ untranslated region and two single nucleotide polymorphisms (SNPs), rs2652511 and rs2937639, in the SLC6A3 gene were genotyped. Among the 38 participants, those with a VNTR other than $10 R / 10 R(n=7)$ were excluded. The alleles of the two SNPs (rs2652511 and rs2937639) appeared to be inherited together in two fixed combinations ( $\mathrm{C}-\mathrm{G}$ or $\mathrm{T}-\mathrm{A}$ ) in 29 of 31 individuals. The $\mathrm{BP}_{\mathrm{ND}}$ in the ventral striatum (VST), caudate nucleus, and putamen was not significantly different after glucose or placebo loading according to genotype. However, $\mathrm{BP}_{\mathrm{ND}}$ s from the caudate nucleus and putamen of all participants with rs 2652511 $\mathrm{CT} / \mathrm{rs} 2937639$ AG $(\mathrm{n}=6)$ were higher after glucose loading. In conclusion, the SLC6A3 gene polymorphism is associated with the changes in DAT availability after glucose loading. DAT availability after glucose or placebo loading in the VST, caudate nucleus, and putamen did not differ according to the SLC6A3 genotype.

\section{Introduction}

The dopamine transporter (DAT) is a transmembrane protein that actively translocates dopamine from the extracellular space into presynaptic neurons in the dopaminergic system (Vaughan and Foster, 2013). Dysfunction of the DAT has been linked to neuropsychiatric disorders, such as attentiondeficit/hyperactivity disorder (Roessner et al., 2010), bipolar disorder (Mick et al., 2008), and alcoholism (Du et al., 2011). Additionally, SLC6A3 gene polymorphisms have been shown to be associated with the expression of striatal DAT (van de Giessen et al., 2009). Among SLC6A3 gene polymorphisms, most previous studies have focused on the 40-base-pair variable number tandem repeat (VNTR) in the $3^{\prime}$ untranslated region of the SLC6A3 gene. According to a meta-analysis by Faraone et al., a 9-repeat (9R) allele of the VNTR is associated with increased DAT availability in the striatum, independent of the presence of neuropsychiatric disorders (Faraone et al., 2014). However, other SLC6A3 gene polymorphisms have rarely been investigated.

The brain plays a major role in regulating the energy balance of the body (van Galen et al., 2018). Eating behavior is a process of energy intake, controlled by both the homeostatic and hedonic systems of the brain (Morton et al., 2014; van Galen et al., 2018). The hypothalamus plays a central role in maintaining the physiologic requirements of the body, while the striatum is the major organ that regulates eating behavior through the reward system (Khanh et al., 2014). Regarding neurotransmitters, dopamine plays an important role in reward processing (Wang et al., 2011). However, the DAT is not considered to be involved in the neurobiology underlying eating behavior in humans (Thomsen et al., 2013). Previously, substantial increases in DAT in response to glucose loading were observed, and an important role of DAT 
in eating behavior was proposed by Pak et al. (Pak et al., 2020). However, not all individuals showed an increase in striatal DAT availability in response to glucose loading (Pak et al., 2020). Therefore, we hypothesized that SLC6A3 polymorphisms underlie the changes in DAT availability after glucose loading in humans.

\section{Materials And Methods}

\section{Participants}

All participants signed an informed consent form prior to participation. Healthy male individuals were included in this study. Heavy smokers; participants with more than a $10 \%$ change in weight over six months; and those with a history of drug abuse, brain injury, neuropsychological disorders, or endocrine disorders were excluded. On the day of each visit, the participants were instructed to fast overnight for at least 12 hours and abstain from smoking and alcohol consumption. The participants visited the institution between 11 am and $12 \mathrm{pm}$ to avoid the effect of diurnal variations in dopamine. Twenty-seven participants in this study were included in a previous study of striatal DAT changes after glucose loading (Pak et al., 2020). This study was approved by the institutional review board of Pusan National University Hospital.

\section{Study design}

Each participant visited the institution two times, on separate days, for two positron emission tomography (PET) scans. Bilateral antecubital veins were cannulated: one for blood sampling and injection of ${ }^{18} \mathrm{~F}-\mathrm{FP}-\mathrm{CIT}$, and the other for glucose or placebo infusions. The participants were blinded and randomly assigned to either glucose or placebo infusions. Over $10 \mathrm{~min}, 300 \mathrm{mg} / \mathrm{kg}$ of glucose in a $50 \%$ solution was administered. The placebo (normal saline) was also administered at the same speed and volume as the $300 \mathrm{mg} / \mathrm{kg}$ of glucose (Haltia et al., 2007). The serum glucose level (mg/dL) and insulin level $(\mu \mathrm{U} / \mathrm{mL})$ were measured before and after the infusion of glucose and placebo. The serum glucose level was determined by using an enzymatic reference method using hexokinase with the Glucose HK Gen.3 (Roche Diagnostics GmbH, Germany). The serum insulin level was determined using an electrochemiluminescence immunoassay method using Elecsys Insulin (Roche Diagnostics $\mathrm{GmbH}$, Germany). An intravenous bolus injection of ${ }^{18} \mathrm{~F}$-FP-CIT $(210.9 \pm 16.3 \mathrm{MBq})$ was administered after the infusion of glucose or placebo. The emission data were acquired over 90 min with 50 frames of progressively increasing durations ( $15 \mathrm{~s} \times 8$ frames, $30 \mathrm{~s} \times 16$ frames, $60 \mathrm{~s} \times 10$ frames, $240 \mathrm{~s} \times 10$ frames, and $300 \mathrm{~s} \times 6$ frames) using Siemens Biograph 40 Truepoint (Siemens Healthcare, Knoxville, Tennessee, USA). The dynamic PET data were collected in 3-dimensional mode, producing with 148 slices with image sizes of $256 \times 256$ and pixel sizes of $1.3364 \times 1.3364 \mathrm{~mm}^{2}$. These were reconstructed using filtered back projection with a Gaussian filter.

\section{Genotyping}


The DNA of each participant was extracted for genotyping from whole-blood samples. The 40-bp VNTR in the 3' untranslated region and two single nucleotide polymorphisms (SNPs), rs2652511 and rs2937639, in the SLC6A3 gene were genotyped (van de Giessen et al., 2009). The primers for the VNTR were adopted from those used in a previous study by Vandenbergh et al. (Vandenbergh et al., 1992), and those for the SNPs were designed using Primer3 (v4.1.0, Whitehead Institute for Biomedical Research, Cambridge, MA, USA)(Koressaar and Remm, 2007; Untergasser et al., 2012). Direct sequencing was performed using the Bigdye Terminator v3.1 Cycle Sequencing Kit (ThermoFisher, MA, USA) and the Applied Biosystems 3730XL DNA Analyzer (ThermoFisher, MA, USA).

\section{Image analysis}

For a volume-of-interest (VOI)-based analysis, an averaged image (0-10 min after injection) was created from dynamic PET frames and spatially normalized to a ${ }^{15} 0$-water PET template in Statistical Parametric Mapping 5 (Wellcome Trust Centre for Neuroimaging, United Kingdom). To extract time-activity curves (TACs) of VOIs from full dynamic PET scans, the Oxford-GSK-Imanova striatal atlas from FMRIB Software Library v5.0 (https://fsl.fmrib.ox.ac.uk/fsl) was applied, which is an atlas of the sub-striatal regions of the ventral striatum (VST), caudate nucleus, and putamen segmented according to the anatomical structure, and manually delineated on the non-linear MNI 152 template (Tziortzi et al., 2011). DAT availability, expressed in terms of binding potential $\left(\mathrm{BP}_{\mathrm{ND}}\right)$, was measured by analyzing TACs via the simplified reference tissue method (Lammertsma and Hume, 1996) using the cerebellum as a reference. Image analysis was performed using pmod version 3.6 (PMOD Technologies LLC, Zurich, Switzerland). For a voxel-based analysis, parametric maps were generated for each participant and analyzed using a paired t-test. Results were displayed at a significance threshold of uncorrected $p<0.0005$ and minimum cluster size for 10 contiguous voxels. Image analysis was performed using SPM12 (Wellcome Trust Centre for Neuroimaging, London, UK) implemented on Matlab R2016b (MathWorks, USA).

\section{Statistical analysis}

Normality was assessed using the D'Agostino \& Pearson normality test. The Mann-Whitney test was used to compare $\mathrm{BP}_{\mathrm{ND}} \mathrm{S}$ from the VST, caudate nucleus, and putamen after placebo and glucose loading according to genotypes. Wilcoxon matched-pairs signed rank test was used to compare $\mathrm{BP}_{\mathrm{ND}}$ after placebo and glucose loading separately according to genotypes. A chi-squared test was used to test the difference of frequency in the increase or decrease in $\mathrm{BP}_{\mathrm{ND}} \mathrm{S}$ after placebo and glucose loading according to genotypes. All analyses were conducted using Prism (v7.0d, GraphPad Software Inc, La Jolla, CA, USA).

\section{Results}

Thirty-eight healthy men aged 20-31 years were enrolled in this study. After excluding individuals with VNTRs other than 10R/10R ( $n=7), 31$ participants were categorized according to the rs2652511 and rs2937639 genotypes. The alleles of the two SNPs (rs2652511 and rs2937639) appeared to be inherited together in two fixed combinations (C-G or T-A) in 29 of 31 participants (rs2652511 CC/rs2937639 GG [n 
= 1]; rs2652511 CT/ rs2937639 AG ( $=$ = 6); rs2652511 TT/rs2937639 AA [n = 23]; rs2652511

TT/rs2937639 GG [n = 1]). To evaluate the effect of rs2652511 and rs2937639 genotypes, 29 participants with rs2652511 CT/rs2937639 AG $(n=6)$ or rs2652511 TT/rs2937639 AA [n= 23] were included in this study. The characteristics of the participants are shown in Table 1.

Table 1

Characteristics of participants

\begin{tabular}{|llll|}
\hline Variables & $\begin{array}{l}\text { rs2652511 CT/rs2937639AG } \\
(\mathbf{n = 6})\end{array}$ & $\begin{array}{l}\text { rs2652511 TT/rs2937639 AA } \\
(\mathbf{n = 2 3})\end{array}$ & $\mathbf{p}$ \\
\hline Age (years) & $24.0 \pm 2.5$ & $24.4 \pm 2.7$ & 0.5626 \\
\hline $\begin{array}{l}\text { Body mass index } \\
\left(\mathrm{kg} / \mathrm{m}^{2}\right)\end{array}$ & $21.9 \pm 2.2$ & $24.1 \pm 3.8$ & 0.1119 \\
\hline $\mathrm{BP}_{\mathrm{ND}}$ & $5.3 \pm 1.5$ & $4.5 \pm 1.6$ & 0.7331 \\
Placebo loading & $2.3 \pm 2.1$ & $3.5 \pm 1.9$ & 0.2777 \\
VST & $4.0 \pm 2.0$ & $5.6 \pm 2.2$ & 0.3841 \\
Caudate nucleus & $4.4 \pm 1.8$ & $4.8 \pm 1.9$ & 0.2582 \\
Putamen & $4.9 \pm 2.0$ & $3.7 \pm 2.0$ & 0.1925 \\
Glucose loading & $6.8 \pm 1.6$ & $5.9 \pm 2.1$ & 0.1894 \\
VST & & \\
Caudate nucleus & & & \\
\hline Putamen & & \\
\hline Data are expressed as mean \pm standard deviation. & & \\
\hline BP & & \\
\hline
\end{tabular}

$\mathrm{BP}_{\mathrm{ND}} \mathrm{S}$ in the VST, caudate nucleus, and putamen were compared between participants with rs 2652511 $\mathrm{CT} / \mathrm{rs} 2937639 \mathrm{AG}$ and rs2652511 TT/rs2937639 AA. BP $\mathrm{ND}_{\text {in }}$ ine VST, caudate nucleus, and putamen was not significantly different after glucose $(p=0.7331, p=0.2777$, and $p=0.3841$, respectively) or placebo ( $p=0.2582, p=0.1925$, and $p=0.1894$, respectively) loading according to the genotype (Fig. 1 ). rs2652511 and rs2937639 genotypes did not have any effect on the changes in $\mathrm{BP}_{\mathrm{ND}} \mathrm{s}$ in the VST ( $\mathrm{p}=$ $\left.0.4190, \chi^{2}=0.653\right)$. However, $\mathrm{BP}_{\mathrm{ND}} \mathrm{s}$ in the caudate nucleus $\left(\mathrm{p}=0.0232, \chi^{2}=5.156\right)$, and putamen $(p=$ $\left.0.0499, \chi^{2}=3.844\right)$ of all participants with rs2652511 CT/rs2937639 AG $(n=6)$ were higher after glucose loading than after placebo loading (Fig. 2). From a voxel-based analysis of participants with rs2652511 CT/rs2937639 AG, significant increases after glucose loading were identified in the right putamen $(x, y, z$, cluster size: 24,2 , 2, 15 voxels), left putamen ( $x, y$, $z$, cluster size: $-26,14,6 ;-28,2,6$; 59 voxels), right 
caudate nucleus ( $x, y, z$, cluster size: 12, 12, 10; 14 - 220; 55 voxels), left caudate nucleus ( $x, y, z$, cluster size: $-16,8,12 ; 12$ voxels) (Fig. 3 ).

\section{Discussion}

In this study, the DAT availability in the VST, caudate nucleus, and putamen did not differ with the SLC6A3 genotype (rs2652511; rs2937639). However, changes in DAT availability after glucose loading were affected by the SLC6A3 genotype (rs2652511; rs2937639). In all participants with rs2652511 CT/rs2937639 AG, striatal DAT availability was increased after glucose loading.

Eating behavior is regulated by both the homeostatic and hedonic systems of the brain (van Galen et al., 2018). The hypothalamus plays a central role in maintaining the physiologic requirements of the body, while the striatum is the major organ that regulates eating behavior through the reward system (Khanh et al., 2014). Among neurotransmitters, dopamine plays a particularly important role in reward processing (Wang et al., 2011). Unlike the dopamine receptor, the DAT, which actively translocates dopamine from the extracellular space into presynaptic neurons (Vaughan and Foster, 2013) was not thought to be involved in the neurobiology underlying obesity in humans (Thomsen et al., 2013) as there was no significant correlation between DAT availability and body mass index (BMI)(Thomsen et al., 2013; van de Giessen et al., 2013; Nam et al., 2018). We have previously highlighted the role of DAT (Pak et al., 2020). In a previous study, substantial increases in DAT availability were observed at least $18 \%$ after glucose loading, although the paired t-test of DAT availability between placebo and glucose loading did not find a significant difference (Pak et al., 2020). In addition, BMI was negatively correlated with DAT availability after glucose loading (Pak et al., 2020). In this regard, participants with a lower BMI may have 1) a higher clearance of synaptic dopamine, and 2) a lower endogenous concentration of dopamine due to higher DAT availability, leading to the stop of eating behavior (Pak et al., 2020). According to Jones et al., insulin activates the PI3K/Akt signaling pathway, enhancing the surface expression of striatal DAT in animal studies (Jones et al., 2017). However, not all participants showed an increase in striatal $\mathrm{BP}_{\mathrm{ND}}$ after glucose loading equally, leading to the insignificant results in paired t-tests (Pak et al., 2020). Therefore, we hypothesized that SLC6A3 gene polymorphisms may affect the changes in DAT availability after glucose loading.

Dysfunction of the DAT has been linked to neuropsychiatric disorders, such as attentiondeficit/hyperactivity disorder (Roessner et al., 2010), bipolar disorder (Mick et al., 2008), and alcoholism (Du et al., 2011). Additionally, SLC6A3 gene polymorphisms have been shown to be associated with the expression of striatal DAT (van de Giessen et al., 2009). Among polymorphisms of the SLC6A3 gene, most previous studies have focused on the 40-base-pair VNTR in the 3' untranslated region of the SLC6A3 gene. According to a meta-analysis by Faraone et al., the 9R allele of the VNTR is associated with increased DAT availability, and the VNTR has an effect on DAT availability (Faraone et al., 2014). In a study by van de Giessen et al. (van de Giessen et al., 2009), 58.4\% (45/77) of the participants had a VNTR of $10 R / 10 R$, while this was present in $81.6 \%(31 / 38)$ of participants included in the current study. Ethnic differences may explain this difference in the proportion of the 10R/10R VNTR in each study group. To 
rule out the effect of the VNTR on DAT availability, only the 31 participants with the 10R/10R VNTR were included in this study. Previously, only two studies investigated the effects of SLC6A3 gene polymorphisms other than the VNTR on DAT availability (Drgon et al., 2006; van de Giessen et al., 2009). Drgon et al. screened and identified SNPs of rs2652511 and rs2937639 at the $5^{\prime}$ end of the SLC6A3 gene (Drgon et al., 2006). rs2652511 is located in the 5 ' flanking sequences of the SLC6A3 gene, while rs2937639 is located in intron 1 of the SLC6A3 gene (van de Giessen et al., 2009). Participants with rs2652511 C/rs2937639 G had more frequent in highly expressed DAT availability with ${ }^{11} \mathrm{C}$-cocaine PET (Drgon et al., 2006). However, in a study by van de Giessen et al., neither rs2652511 nor rs2937639 was associated with striatal DAT availability as measured using ${ }^{123} \mathrm{I}-\beta$-CIT SPECT (van de Giessen et al., 2009), consistent with the findings of the present study. As we discovered the changes in DAT after glucose loading in humans (Pak et al., 2020), we investigated the association between SLC6A3 genotypes (rs2652511; rs2937639) and DAT availability after glucose loading. However, the SLC6A3 genotype did not have an effect on DAT availability after glucose loading. Consistent with two previous studies, rs2652511 and rs2937639 were inherited as fixed allele combinations in 29 of 31 participants (93.5\%); either rs2652511 CT/rs2937639 AG $(n=6)$ or rs2652511 TT/rs2937639 AA $(n=23)$. In all participants with rs2652511 CT/rs2937639 AG, DAT availability in the dorsal striatum (caudate nucleus and putamen) increased after glucose loading, whereas they were increased in $47.8 \%$ (caudate nucleus) and $56.5 \%$ (putamen) of participants with rs2652511 TT/rs2937639 AA. Therefore, SLC6A3 gene polymorphisms (rs2652511; rs2937639) may affect changes in DAT availability after glucose loading according to this preliminary study. However, the changes in DAT availability in the VST were not affected by the SLC6A3 genotype. The VST and dorsal striatum (caudate nucleus and putamen) are known to have distinct roles. The VST plays a major role in processing reward cues and in the motivation to seek rewards (Caravaggio et al., 2015), while the dorsal striatum (caudate nucleus and putamen) is involved in non-hedonic food motivations of caloric requirements for survival. Therefore, region-dependent DAT regulatory mechanisms (ventral vs. dorsal striatum) might exist in the response to glucose loading reflecting eating behavior.

This study has some limitations. A small number of participants were included in the study. To validate this preliminary study, further studies with larger numbers of participants are needed. To consider the effect of rs2652511 and rs2937639 genotypes, individuals with a VNTR other than 10R/10R were excluded from this study. In addition, a screening procedure might be needed to investigate other candidates of SLC6A3 gene polymorphisms that have an effect on DAT availability.

In conclusion, we have highlighted that the SLC6A3 gene polymorphism is associated with the changes in DAT availability after glucose loading. However, DAT availability after glucose or placebo loading in the VST, caudate nucleus, and putamen did not differ according to the SLC6A3 genotype. Further studies with a larger number of participants are needed to validate this finding.

\section{Abbreviations}

DAT, dopamine transporter 
$\mathrm{BP}_{\mathrm{ND}}$, binding potential

SNP, single nucleotide polymorphisms

PET, positron emission tomography

VST, ventral striatum

\section{Declarations}

FUNDING

No

\section{CONFLICTS OF INTERESTS}

The authors declare that they have no competing interests.

\section{AVAILABILITY OF DATA AND MATERIALS}

All data are available to corresponding author of the manuscript upon reasonable request.

\section{AUTHOR's CONTRIBUTIONS}

Kyoungjune Pak; study design, write the manuscript

Seongho Seo; image analysis

Keunyoung Kim; image analysis

Myung Jun Lee; image analysis, study design

Seong Janng Kim: image analysis

In Joo Kim; write the manuscript

\section{Ethics approval and consent to participate}

All procedures performed in studies involving human participants were in accordance with the ethical standards of the institutional and/or national research committee and with the 1964 Declaration of Helsinki and its later amendments or comparable ethical standards. Ethical permission for the study procedures was obtained from the Institutional Review Boards at Pusan National University Hospital. 


\section{Consent to participate}

Subject consent has been obtained by Pusan National University Hospital.

\section{Consent for publication}

Not applicable.

\section{References}

1. Caravaggio, F., Raitsin, S., Gerretsen, P., Nakajima, S., Wilson, A. \& Graff-Guerrero, A. (2015) 'Ventral striatum binding of a dopamine D2/3 receptor agonist but not antagonist predicts normal body mass index', Biol Psychiatry, 77(2), pp. 196-202.

2. Drgon, T., Lin, Z., Wang, G. J., Fowler, J., Pablo, J., Mash, D. C., Volkow, N. \& Uhl, G. R. (2006) 'Common human 5 ' dopamine transporter (SLC6A3) haplotypes yield varying expression levels in vivo', Cell Mol Neurobiol, 26(4-6), pp. 875-889.

3. Du, Y., Nie, Y., Li, Y. \& Wan, Y. J. (2011) 'The association between the SLC6A3 VNTR 9-repeat allele and alcoholism-a meta-analysis', Alcohol Clin Exp Res, 35(9), pp. 1625-1634.

4. Faraone, S. V., Spencer, T. J., Madras, B. K., Zhang-James, Y. \& Biederman, J. (2014) 'Functional effects of dopamine transporter gene genotypes on in vivo dopamine transporter functioning: a meta-analysis', Mol Psychiatry, 19(8), pp. 880-889.

5. Haltia, L. T., Rinne, J. O., Merisaari, H., Maguire, R. P., Savontaus, E., Helin, S., Nagren, K. \& Kaasinen, V. (2007) 'Effects of intravenous glucose on dopaminergic function in the human brain in vivo', Synapse, 61(9), pp. 748-756.

6. Jones, K. T., Woods, C., Zhen, J., Antonio, T., Carr, K. D. \& Reith, M. E. (2017) 'Effects of diet and insulin on dopamine transporter activity and expression in rat caudate-putamen, nucleus accumbens, and midbrain', J Neurochem, 140(5), pp. 728-740.

7. Khanh, D. V., Choi, Y. H., Moh, S. H., Kinyua, A. W. \& Kim, K. W. (2014) 'Leptin and insulin signaling in dopaminergic neurons: relationship between energy balance and reward system', Front Psychol, 5, p. 846.

8. Koressaar, T. \& Remm, M. (2007) 'Enhancements and modifications of primer design program Primer3', Bioinformatics, 23(10), pp. 1289-1291.

9. Lammertsma, A. A. \& Hume, S. P. (1996) 'Simplified reference tissue model for PET receptor studies', Neuroimage, 4(3 Pt 1), pp. 153-158.

10. Mick, E., Kim, J. W., Biederman, J., Wozniak, J., Wilens, T., Spencer, T., Smoller, J. W. \& Faraone, S. V. (2008) 'Family based association study of pediatric bipolar disorder and the dopamine transporter gene (SLC6A3)', Am J Med Genet B Neuropsychiatr Genet, 147B(7), pp. 1182-1185. 
11. Morton, G. J., Meek, T. H. \& Schwartz, M. W. (2014) 'Neurobiology of food intake in health and disease', Nat Rev Neurosci, 15(6), pp. 367-378.

12. Nam, S. B., Kim, K., Kim, B. S., Im, H. J., Lee, S. H., Kim, S. J., Kim, I. J. \& Pak, K. (2018) 'The Effect of Obesity on the Availabilities of Dopamine and Serotonin Transporters', Sci Rep, 8(1), p. 4924.

13. Pak, K., Seo, S., Kim, K., Lee, M. J., Shin, M. J., Suh, S., Im, H. J., Park, J. J., Kim, S. J. \& Kim, I. J. (2020) 'Striatal dopamine transporter changes after glucose loading in humans', Diabetes Obes Metab, 22(1), pp. 116-122.

14. Roessner, V., Sagvolden, T., Dasbanerjee, T., Middleton, F. A., Faraone, S. V., Walaas, S. I., Becker, A., Rothenberger, A. \& Bock, N. (2010) 'Methylphenidate normalizes elevated dopamine transporter densities in an animal model of the attention-deficit/hyperactivity disorder combined type, but not to the same extent in one of the attention-deficit/hyperactivity disorder inattentive type', Neuroscience, 167(4), pp. 1183-1191.

15. Thomsen, G., Ziebell, M., Jensen, P. S., da Cuhna-Bang, S., Knudsen, G. M. \& Pinborg, L. H. (2013) 'No correlation between body mass index and striatal dopamine transporter availability in healthy volunteers using SPECT and [123I]PE2I', Obesity (Silver Spring), 21(9), pp. 1803-1806.

16. Tziortzi, A. C., Searle, G. E., Tzimopoulou, S., Salinas, C., Beaver, J. D., Jenkinson, M., Laruelle, M., Rabiner, E. A. \& Gunn, R. N. (2011) 'Imaging dopamine receptors in humans with [11C]-(+)-PHNO: dissection of D3 signal and anatomy', Neuroimage, 54(1), pp. 264-277.

17. Untergasser, A., Cutcutache, I., Koressaar, T., Ye, J., Faircloth, B. C., Remm, M. \& Rozen, S. G. (2012) 'Primer3--new capabilities and interfaces', Nucleic Acids Res, 40(15), p. e115.

18. van de Giessen, E., de Win, M. M., Tanck, M. W., van den Brink, W., Baas, F. \& Booij, J. (2009) 'Striatal dopamine transporter availability associated with polymorphisms in the dopamine transporter gene SLC6A3', J Nucl Med, 50(1), pp. 45-52.

19. van de Giessen, E., Hesse, S., Caan, M. W., Zientek, F., Dickson, J. C., Tossici-Bolt, L., Sera, T., Asenbaum, S., Guignard, R., Akdemir, U. O., Knudsen, G. M., Nobili, F., Pagani, M., Vander Borght, T., Van Laere, K., Varrone, A., Tatsch, K., Booij, J. \& Sabri, O. (2013) 'No association between striatal dopamine transporter binding and body mass index: a multi-center European study in healthy volunteers', Neuroimage, 64, pp. 61-67.

20. van Galen, K. A., Ter Horst, K. W., Booij, J., la Fleur, S. E. \& Serlie, M. J. (2018) 'The role of central dopamine and serotonin in human obesity: lessons learned from molecular neuroimaging studies', Metabolism, 85, pp. 325-339.

21. Vandenbergh, D. J., Persico, A. M., Hawkins, A. L., Griffin, C. A., Li, X., Jabs, E. W. \& Uhl, G. R. (1992) 'Human dopamine transporter gene (DAT1) maps to chromosome 5p15.3 and displays a VNTR', Genomics, 14(4), pp. 1104-1106.

22. Vaughan, R. A. \& Foster, J. D. (2013) 'Mechanisms of dopamine transporter regulation in normal and disease states', Trends Pharmacol Sci, 34(9), pp. 489-496.

23. Wang, G. J., Geliebter, A., Volkow, N. D., Telang, F. W., Logan, J., Jayne, M. C., Galanti, K., Selig, P. A., Han, H., Zhu, W., Wong, C. T. \& Fowler, J. S. (2011) 'Enhanced striatal dopamine release during food 


\section{Figures}
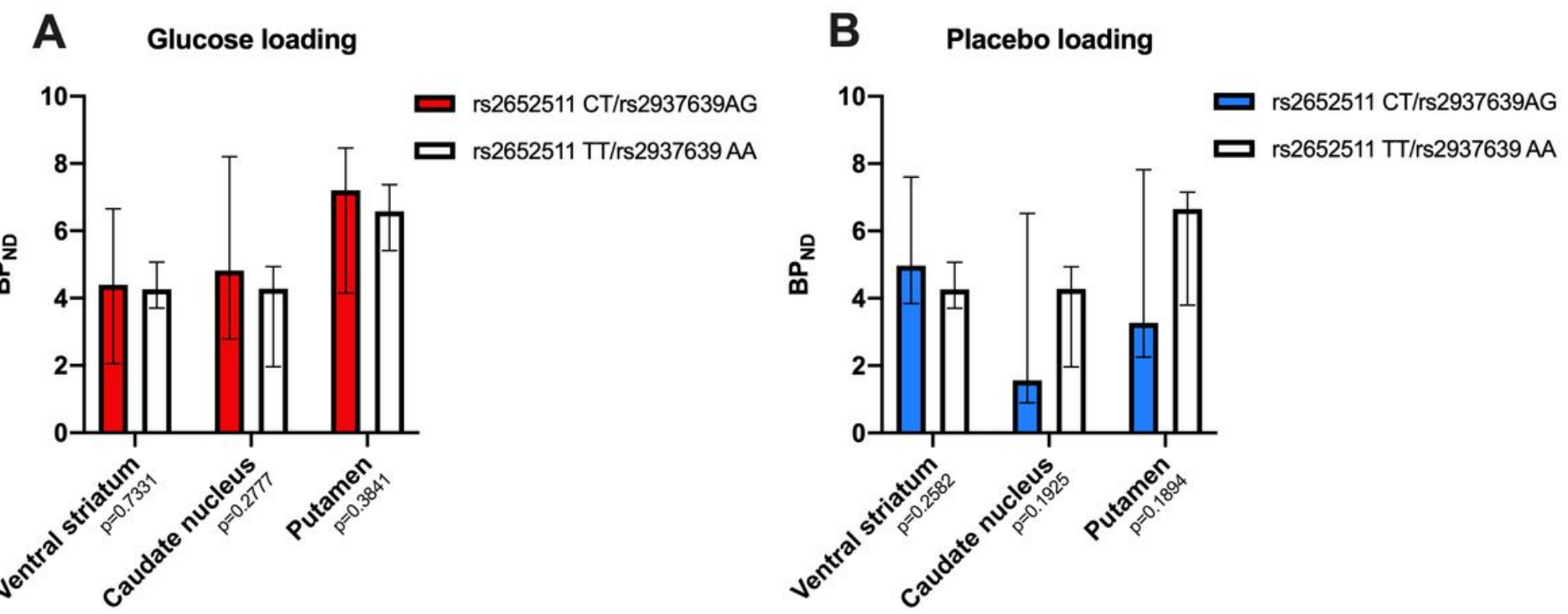

Figure 1

BPND (binding potential) after glucose (A) and placebo (B) loading according to genotype
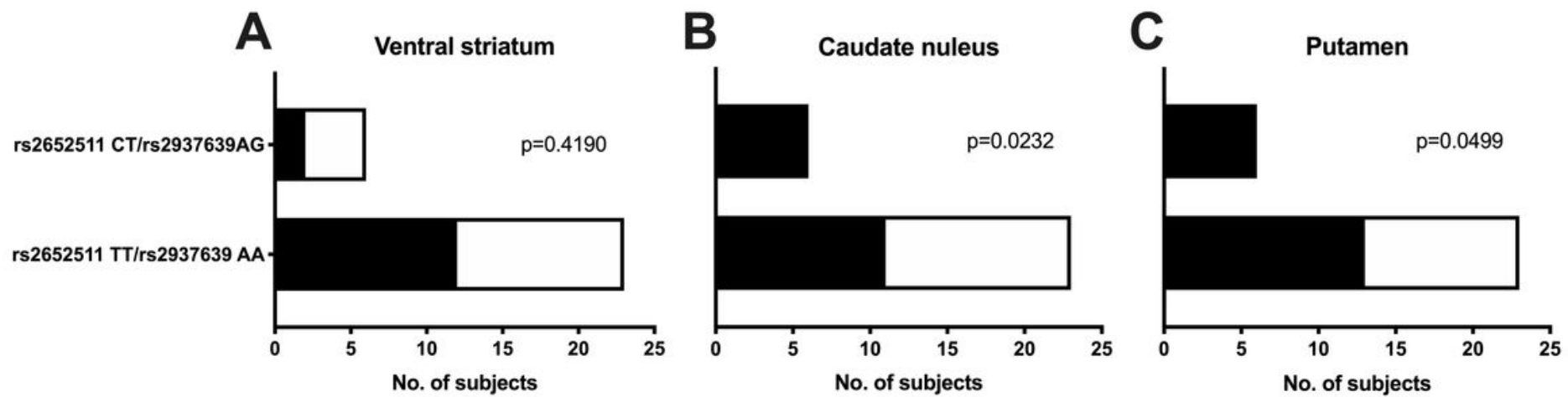

Figure 2

Changes in BPND (binding potential) in the ventral striatum (A), caudate nucleus (B), and putamen (C) after glucose and placebo loading according to genotype 

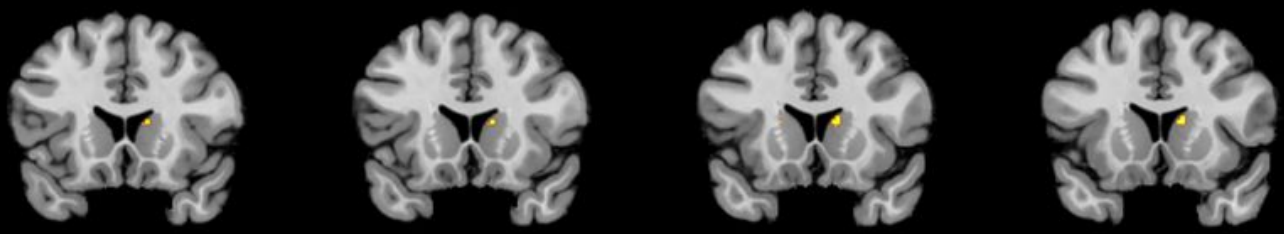

$$
y=18
$$
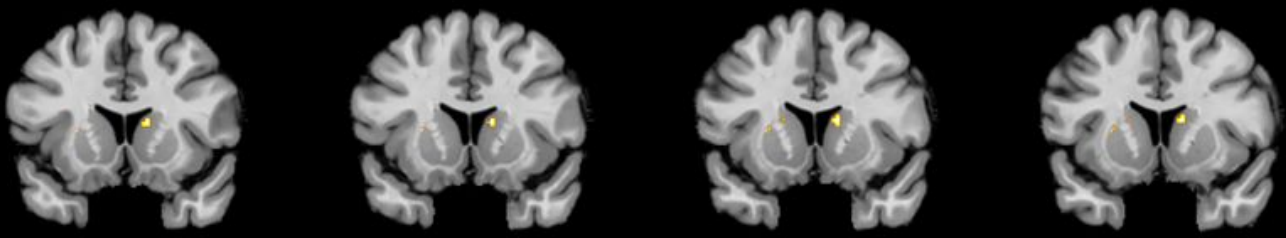

$y=14$
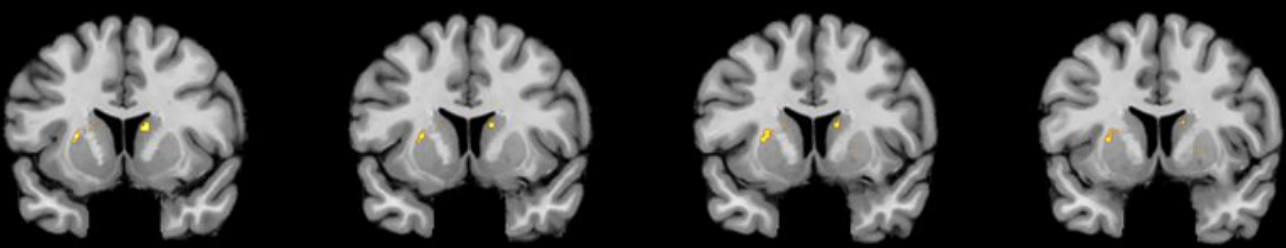

$$
y=10
$$
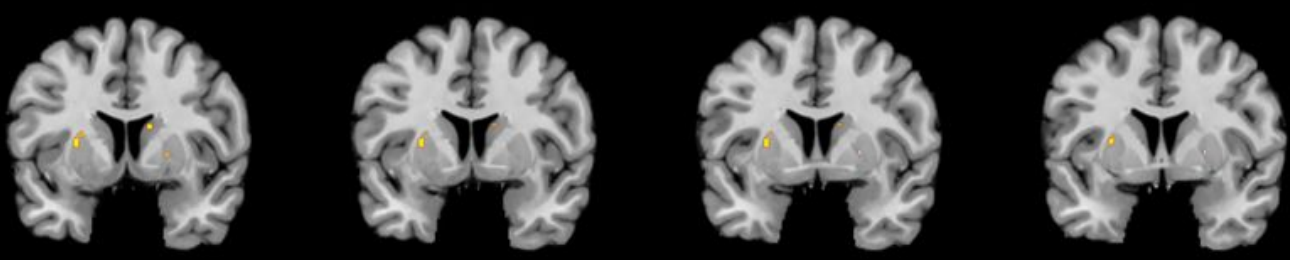

$$
y=6
$$
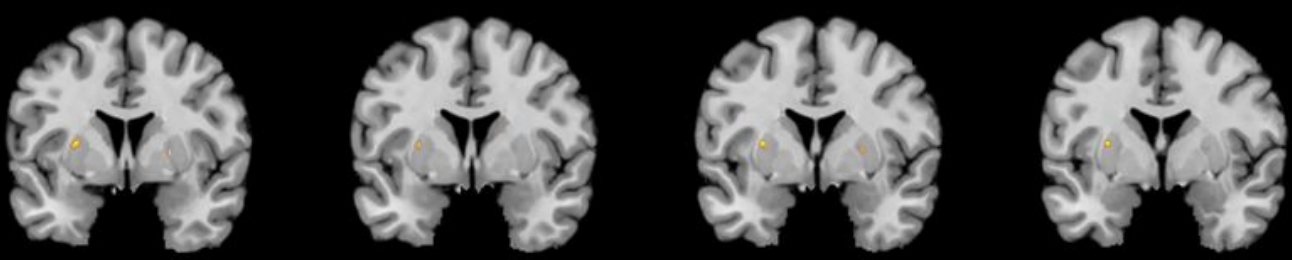

$$
y=2
$$

\section{\begin{tabular}{ll|l}
0 & $\mathrm{z}$ & 9
\end{tabular}}

Figure 3

Areas of increased BPND (binding potential) after glucose loading in participants with rs2652511 CT/rs2937639 AG 